\title{
Response Monitoring, Tolerability, and Effectiveness of Imatinib Treatment for Chronic Myeloid Leukemia in a Retrospective Research Database
}

\author{
David D. Stenehjem, PharmD ${ }^{\mathrm{a}, \mathrm{b}}$; Frederick Albright, $\mathrm{PhD}^{\mathrm{a}}$; Kuan-Ling Kuo, BPharm; \\ Karina Raimundo, MS ${ }^{\mathrm{a}, \mathrm{c}}$; Hillevi Bauer, BPharma ; Paul J. Shami, MD'; Michael W. Deininger, MD, PhD;; \\ Lei Chen, $\mathrm{MD}, \mathrm{PhD}^{c}$; and Diana I. Brixner, $\mathrm{PhD}, \mathrm{RPh}^{\mathrm{a}, \mathrm{e}}$
}

\begin{abstract}
Retrospective review of imatinib monitoring through electronic health records (EHR) can provide valuable insight into the current management of chronic myelogenous leukemia (CML). This study retrospectively reviewed EHRs from 2001 to 2010 of patients with chronic phase CML (CP-CML) treated with first-line imatinib. Chart evaluations included a review of cytogenetic and molecular testing, overall survival, adverse drug events (ADEs), and therapy modifications. A total of 54 patients with CP-CML were treated with first-line imatinib and had either cytogenetic or molecular testing within 18 months of imatinib initiation. Within the first 18 months of treatment, 33 of 45 patients $(73 \%)$ undergoing cytogenetic testing experienced a complete cytogenetic response
\end{abstract}

From the apepartment of Pharmacotherapy, University of Utah, and 'Huntsman Cancer Institute, University of Utah Health Care, Salt Lake City, Utah; 'Novartis Pharmaceuticals, East Hanover, New Jersey; and the dDivision of Hematology and Hematologic Malignancies, Huntsman Cancer Institute, University of Utah Health Care, and eUniversity of Utah Program in Personalized Health Care, Salt Lake City, Utah.

Submitted July 17, 2013; accepted for publication March 14, 2014. This work was supported by an unrestricted research grant by Novartis Pharmaceuticals. Partial support for all datasets within the Utah Population Database was provided by Huntsman Cancer Institute at the University of Utah. Drs. Stenehjem, Albright, and Brixner, Ms. Kuo, and Ms. Bauer were all partially paid through the University of Utah, by an unrestricted research grant from Novartis to conduct this work. Miss Raimundo receives support from Novartis Pharmaceuticals Corporation. Dr. Shami receives honoraria from Novartis Pharmaceuticals Corporation and Ariad Pharmaceuticals Inc. Dr. Deininger is a consultant for and serves on the advisory board on Bristol-Myers Squibb, Novartis Pharmaceuticals Corporation, and Ariad Pharmaceuticals Inc.; receives honoraria from Bristol-Myers Squibb, Ariad Pharmaceuticals Inc., and Novartis Pharmaceuticals Corporation and receives research funding from Bristol-Myers Squibb, Novartis Pharmaceuticals Corporation, Celgene, and Gilead. Dr. Chen is an Associate Director at and owns stock in Novartis Pharmaceuticals Corporation. A portion of this study was presented at the ASCO Annual Meeting on June 4th, 2012.

Correspondence: Diana I. Brixner, PhD, RPh, Department of Pharmacotherapy, University of Utah College of Pharmacy, 30 South 2000 East, Room 258, Salt Lake City, UT 84112-5820.

E-mail: diana.brixner@utah.edu (median, 241 days; range, 110-542 days) and 24 of 48 patients $(50 \%)$ receiving molecular testing achieved at least a major molecular response (median, 253 days; range, 99-546 days). The average number of cytogenetic and molecular tests conducted within the first 18 months was 2.5 and 3.8, respectively. Nineteen of 54 (35\%) had a dose increase of imatinib (>400 mg; median, 329 days; range, 21-1968 days). The 5-year estimated overall survival rate was $88.5 \%$. Between 2006 and $2010(n=30 ; 56 \%), 7$ patients $(23 \%)$ transitioned to dasatinib or nilotinib (median, 399 days from diagnosis; range, 180-1046 days) because of suboptimal response or treatment failure $(n=5)$ and imatinib $\operatorname{ADEs}(n=2)$. Forty-six imatinib-associated ADEs occurred in 31 patients (57\%), of which 10 $(32 \%)$ received dose reductions (median, 52 days) and $6(19 \%)$ had discontinuations (median, 139 days). Closely monitored patients with CML treated with imatinib at an NCCN Member Institution experienced outcomes comparable to those reported in key clinical trials. (J Natl Compr Canc Netw 2014;12:1113-1121)

Imatinib became the front-line treatment for patients with chronic-phase chronic myelogenous leukemia (CP-CML) based on results from the International Randomized Study of Interferon and STI571 (IRIS), which compared imatinib versus interferon- $\alpha$ combined with low-dose cytarabine. ${ }^{1}$ According to the 5-year follow-up of the IRIS trial, imatinib used as initial therapy produced a cumulative complete cytogenetic response (CCyR) rate of $87 \% .^{1,2}$ The 8-year follow-up, presented in abstract form only, revealed estimated overall and event-free survivals of $85 \%$ and $81 \%$, respectively. ${ }^{3} \mathrm{~Pa}$ tients receiving imatinib have also reported improved health-related quality of life. ${ }^{4}$ However, approximately $30 \%$ of the patients develop resistance and/or intolerance to imatinib. ${ }^{5}$ The second-generation tyrosine kinase inhibitors (TKIs) dasatinib and nilotinib have in- 
creased potency compared with imatinib. ${ }^{6,7}$ They were approved initially for patients in whom imatinib had failed, and subsequently for front-line treatment of patients with newly diagnosed CML. More recently, another second-generation TKI, bosutinib and the third-generation TKI ponatinib, were approved for use in patients for whom previous TKI therapy failed. ${ }^{8,9}$

With the availability of several approved TKIs and the ongoing development of additional therapies, providers are weighing the varying efficacies and toxicities among TKIs in managing their patients with CML. ${ }^{1,2,10}$ Optimal patient management requires comprehensive response monitoring using cytogenetic and molecular testing, along with the assessment of potential toxicities associated with each agent. Toxicity is critical because it may reduce regimen adherence, which in turn is associated with a higher failure rate. ${ }^{11-13}$ The current NCCN Clinical Practice Guidelines in Oncology (NCCN Guidelines) for CML recommend bone marrow cytogenetic testing at diagnosis, at 3 and 6 months if quantitative PCR (international scale [IS]) is not available, and at 12 and 18 months if the patient is not experiencing CCyR or major molecular response (MMR) (to view the most recent version of these guidelines, visit NCCN.org). ${ }^{2,14}$ Quantification of BCR-ABL1 transcripts using quantitative PCR standardized to the IS is recommended before initiation of treatment and at 3-month intervals for 3 years, and every 3 to 6 months thereafter. ${ }^{4,14} \mathrm{Re}$ cently, the NCCN Guidelines were updated to recommend consideration of transition to an alternate TKI if BCR-ABL1 transcripts are greater than 10\% according to quantitative PCR at 3 months. ${ }^{1,5,14}$ This recommendation is based on the results by Marin et al, ${ }^{15}$ suggesting that transcript levels greater than $10 \%$ at 3 months are predictive of poor response to imatinib, and that this finding allows for early intervention to potentially improve outcomes. The goal of this study was to analyze current practice patterns for monitoring response to imatinib and how adverse drug events (ADEs) affect treatment in patients with CML at an NCCN Member Institution.

\section{Methods}

\section{Study Cohort}

The study was based on an observational, cross-sectional cohort of patients with CP-CML treated with first-line imatinib from 2001 to 2010. The index date was defined as the date of CML diagnosis. Inclusion criteria included patients older than 18 years at diagnosis between 2001 and 2010; an ICD-9 code indicative of CML (205.1, 205.10, 205.11, 205.12) and physician or laboratory-documented CP-CML; and use of imatinib as first-line CML therapy (initial cytoreduction with hydroxyurea was allowed). Patients were required to have at least 1 cytogenetic or molecular test from 90 days before diagnosis to 18 months after initiation of imatinib included in electronic data warehouse (EDW) for the University of Utah Health Care (UUHC), which includes Huntsman Cancer Institute (HCI). All diagnoses were confirmed through cytogenetic testing or BCRABL1-positive laboratory results, and/or through either manual chart review or using an automated text searching tool to scan the electronic health record (EHR). CML phase at diagnosis was determined based on EHR review of physician notes, and only patients in $\mathrm{CP}$ at diagnosis were included. Comorbidities at diagnosis were determined by Charlson Comorbidity Index using the classification method developed by D'Hoore et al. ${ }^{16}$ Patients were excluded if the date of diagnosis, phase at diagnosis, or initial CML treatments were not recorded in the EHR. Patients were followed from 90 days before the index date until December 31, 2010; death; or loss to follow-up, whichever came first. The University of Utah Institutional Review Board and the HCI Clinical Cancer Investigational Committee approved this study.

\section{Electronic Data Sources}

The University of Utah Health Sciences EDW ${ }^{17-19}$ and the Utah Population Database (UPDB) ${ }^{20-25}$ served as the primary data sources for this study. All cancer cases across UUHC and HCI are integrated into the EDW, which contains a comprehensive EHR for all patients. ${ }^{17}$ Death certificate and cause of death information were provided by the UPDB originating from the Utah Department of Health Office of Vital Records and Statistics. ${ }^{23,24}$ Patients were identified between these resources by a common master subject index unique for each patient.

\section{Cytogenetic and Molecular Test Results}

Chart review was used to evaluate use and outcome of cytogenetic and molecular testing from 90 days before CML diagnosis until 18 months after initia- 
Monitoring and Tolerance of Imatinib in CML

tion of imatinib and until the end of the follow-up period. Pathology reports for cytogenetic test results and BCR-ABL1 qualitative and quantitative molecular test results were extracted from the EDW, and the quantitative results were converted to the IS based on a laboratory-specific conversion factor. CCyR was defined as no Philadelphia chromosomepositive cells according to chromosome analysis of at least 20 metaphases or according to fluorescence in situ hybridization of at least 50 interphases, and an MMR was defined as less than 0.1\% BCR-ABL1 transcripts by IS.

\section{Adverse Drug Events}

Chart review was performed to determine ADEs and therapy modifications. ADEs of any grade associated with imatinib were documented if a physician correlated the ADE with imatinib.

\section{Therapy Modifications}

Imatinib dose modifications were defined as a dose increase or decrease compared with the starting dose. Reasons for dose modifications were characterized in relation to an ADE, suboptimal response, or treatment failure. Imatinib discontinuation was defined as a change to an alternative therapy.

\section{End Points}

The end points of this study included the average number of cytogenetic or molecular tests conducted from 90 days before diagnosis to 18 months after initiation of imatinib and at any time during followup. The mean number of molecular and cytogenetic tests was also assessed according to year of diagnosis. CML treatment end points included CCyR and MMR within 18 months of imatinib initiation and at any time during follow-up, and overall survival. The frequency and timing of ADEs, and therapy modifications were also assessed.

\section{Statistical Analyses}

Descriptive statistics, including mean, standard deviation, frequency, and percentage for continuous and categorical variables, were used to describe demographic characteristics and comorbidities. Demographic data, drug treatments, and comorbid conditions for patients with CML were collected within 90 days of CML diagnosis. Time-to-event estimates were calculated from the initiation of imatinib using the Kaplan-Meier method using mortality data from the UPDB, and CCyR and MMR data from the
EDW. Data were censored on the last date of followup or on December 31, 2010, whichever occurred earlier. All statistical tests were performed at an a priori significance level of 0.05 using Stata SE, Version 10 (StataCorp, College Station, TX).

\section{Results}

A total of 123 patients were confirmed to have CPCML diagnosed between 2001 and 2010 (Figure 1). Of these, 92 patients $(74.8 \%)$ received imatinib as first-line therapy. A total of 54 (43.9\%) patients receiving cytogenetic or molecular testing from around the time of diagnosis to within 18 months of initiation of imatinib were included in the study. The median age of the study population was 40 years $(\mathrm{SD}$, 17.7) (Table 1). Subjects were more frequently male $(64.8 \%)$, Caucasian $(74.1 \%)$, from the state of Utah $(66.7 \%)$, and had a comorbidity score of 0 (33.3\%) according to the Charlson Comorbidity Index analysis. The median follow-up time was 3.0 years (range, 7 days to 8.1 years; SD, 2.3 years). Of the 38 patients treated with imatinib who did not have cytogenetic or molecular testing within 18 months of diagnosis, $22(57.9 \%)$ were not being treated at the UUHC or HCI for CML, 11 (28.9\%) presented to the institution for CML treatment longer than 18 months after initiating imatinib, $4(10.5 \%)$ were monitored at an outside institution, and 1 (2.6\%) was not monitored because of physician discretion. Four deaths occurred during the study period in the 38 imatinib-treated patients $(10.5 \%)$ who did not have cytogenetic or molecular testing.

\section{Cytogenetic and Molecular Testing}

A total of 153 cytogenetic tests were conducted in 48 patients at any time during follow-up, and 114 $(74.5 \%)$ of these tests were performed in 45 patients from around the time of diagnosis to 18 months after initiation of imatinib (Figure 1). A total of 444 molecular tests were conducted at any time during follow-up in 51 patients, and $182(41.0 \%)$ of these tests were performed in 48 patients from around the time of diagnosis to 18 months after initiation of imatinib. In monitored patients, the average number of cytogenetic tests conducted up to 18 months after starting imatinib was 2.5 (range, 1-7; SD, 1.5), and at any time during a median follow-up of 3.17 years the number of tests per patient was 3.2 (range, 1-10; SD, 2.7) (Figure 1). The average number of 


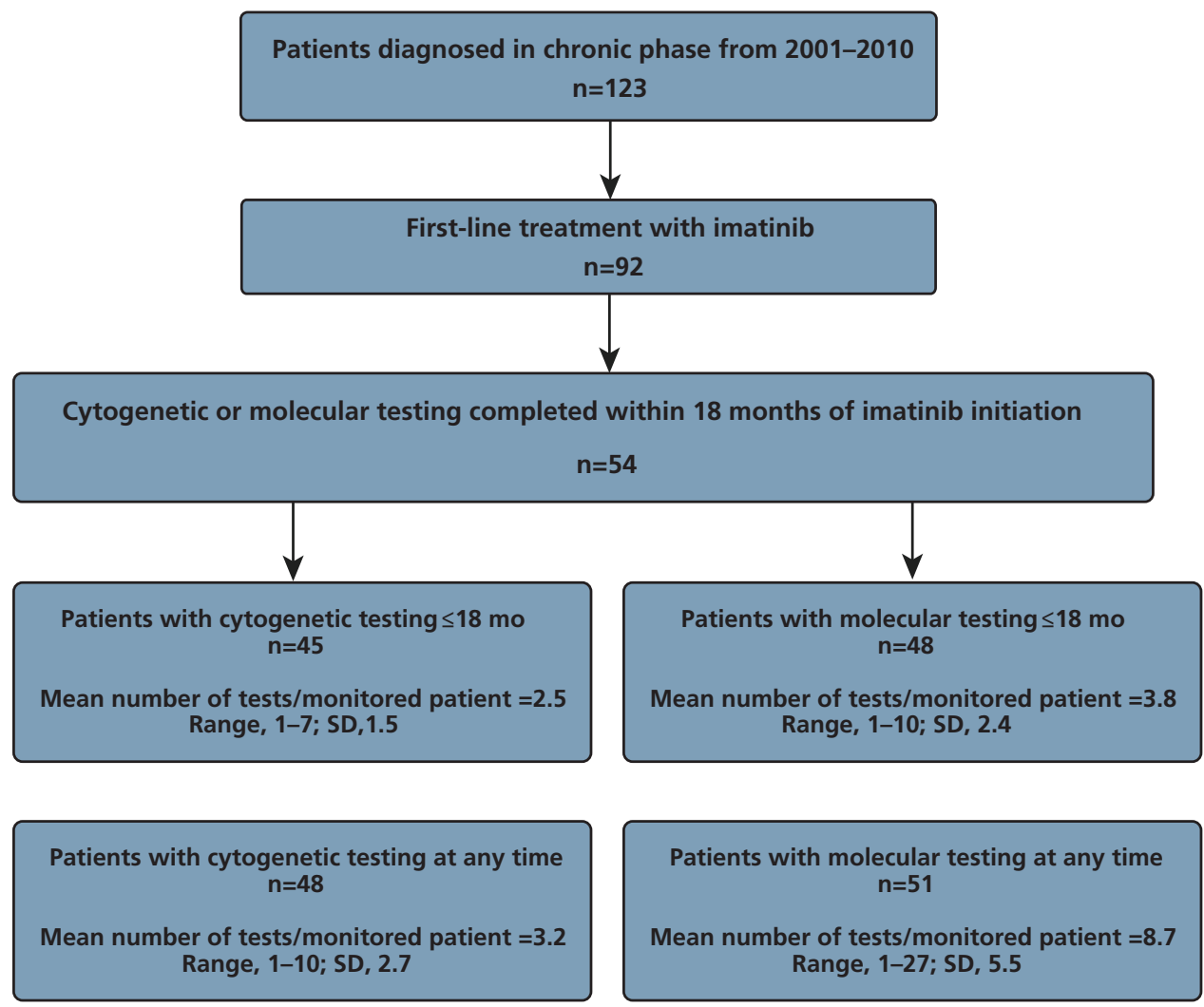

Figure 1 Study cohort and number of cytogenetic and molecular tests conducted per patient.

molecular tests completed in monitored patients up to 18 months after starting imatinib was 3.8 (range, $1-10 ; \mathrm{SD}, 2.4)$, and at any time during a median follow-up of 3.14 years, the number of tests completed increased to 8.7 (range, $1-27$; SD, 5.5). The distribution and timing of all cytogenetic and molecular tests ordered for the cohort are presented in Figure 2. The mean number of cytogenetic and molecular tests conducted from diagnosis to 18 months after starting imatinib per patient by year of diagnosis is presented in Figure 3. Approximately 2 cytogenetic tests were performed per patient diagnosed in 2001-2004 and 3 to 4 cytogenetic tests per patient diagnosed in 2005-2008 were performed from diagnosis to 18 months after starting imatinib. The mean number of molecular tests conducted from diagnosis to 18 months after initiation of imatinib was approximately 1 test per patient diagnosed in 2001, 4 tests per patient diagnosed in 2002-2006, and 5 to 6 tests in patients diagnosed in 2007 and 2008.

Of the 48 patients who were cytogenetically tested at any time, $40(83.3 \%)$ achieved a CCyR at any time, compared with $73 \%$ of those tested within
18 months (Figure 4). Of the 51 patients receiving BCR-ABL1 PCR testing at any time, 37 (72.5\%) achieved at least an MMR at any time compared with $50 \%$ of those tested within 18 months. Of those experiencing a CCyR or MMR within 18 months, the median time to achieving each milestone was 241 days (range, 110-542 days) and 254 days (range, 99-546 days), respectively. For all patients achiev-

\begin{tabular}{|c|c|c|}
\hline & \multicolumn{2}{|c|}{ Imatinib $(\mathrm{N}=54)$} \\
\hline & $\mathbf{N}$ & $\mathrm{N} \%$ \\
\hline Age, y (median, SD) & $40.0 \pm 17.7$ & \\
\hline Sex (male) & 35 & $64.8 \%$ \\
\hline \multicolumn{3}{|l|}{ Race } \\
\hline Caucasian & 40 & $74.1 \%$ \\
\hline Others & 10 & $18.5 \%$ \\
\hline Unknown & 4 & $7.4 \%$ \\
\hline Region (Utah) & 36 & $66.7 \%$ \\
\hline $\begin{array}{l}\text { Charlson Comorbidity } \\
\text { Index (mean, SD) }\end{array}$ & $1.8 \pm 1.8$ & \\
\hline
\end{tabular}


Monitoring and Tolerance of Imatinib in CML

ing a CCyR or MMR at any time, the median time to achieving each milestone was 288.5 days (range, 110-1127 days) and 470 days (range, 99-1785 days), respectively. Kaplan-Meier estimates of the median time to first CCyR and MMR in all patients with at least 1 cytogenetic or molecular test was 13.0 and 18.9 months, respectively (Figure 5). The time to first MMR in patients with a documented ADE was 21.7 months compared with 15.7 months for those without an ADE (nonsignificant; $P=.1535$; Figure 6).

\section{Overall Survival}

The estimated 5-year overall survival rate was $88.5 \%$. Six deaths occurred during the study period, and 3 of these were CML-related, as indicated on their death certificate. Of the deceased patients, 4 (67\%) experienced progression to blast crisis, of which 3 (75\%) underwent allogeneic stem cell transplant; 1 additional patient underwent transplant without history of progression to accelerated or blast phase.

A
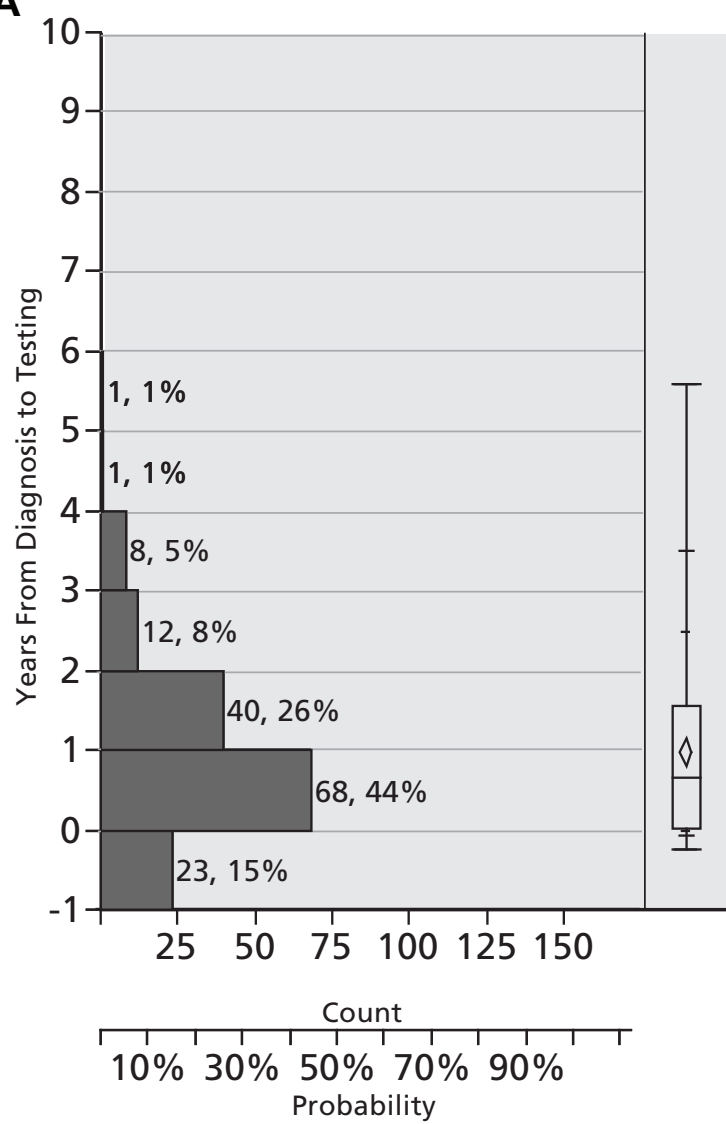

\section{Imatinib-Associated ADEs and}

\section{Therapy Modifications}

Imatinib-associated ADEs of any grade were observed in 31 patients (57\%), and a total of 46 ADEs were observed in these patients. The most commonly reported ADEs were cytopenias $(n=15 ; 33 \%)$, gastrointestinal disturbances $(n=13 ; 30 \%)$, and rash $(n=6 ; 13 \%)$. Other reported ADEs included myalgias, fatigue, fever, edema, and headache. Among patients experiencing an ADE, 10 (32\%) had a dose reduction. The median time to dose reduction was 52 days, and the new median dose was $300 \mathrm{mg} / \mathrm{d}$. A total of 6 patients (19\%) discontinued imatinib because of an ADE, with a median time to discontinuation of 139 days (range, 47-1468 days).

\section{Therapy Modifications for Suboptimal Response or Treatment Failure}

In 19 of the 54 patients (35\%), the imatinib dose was increased to a median dose of $600 \mathrm{mg}$ for suboptimal

B

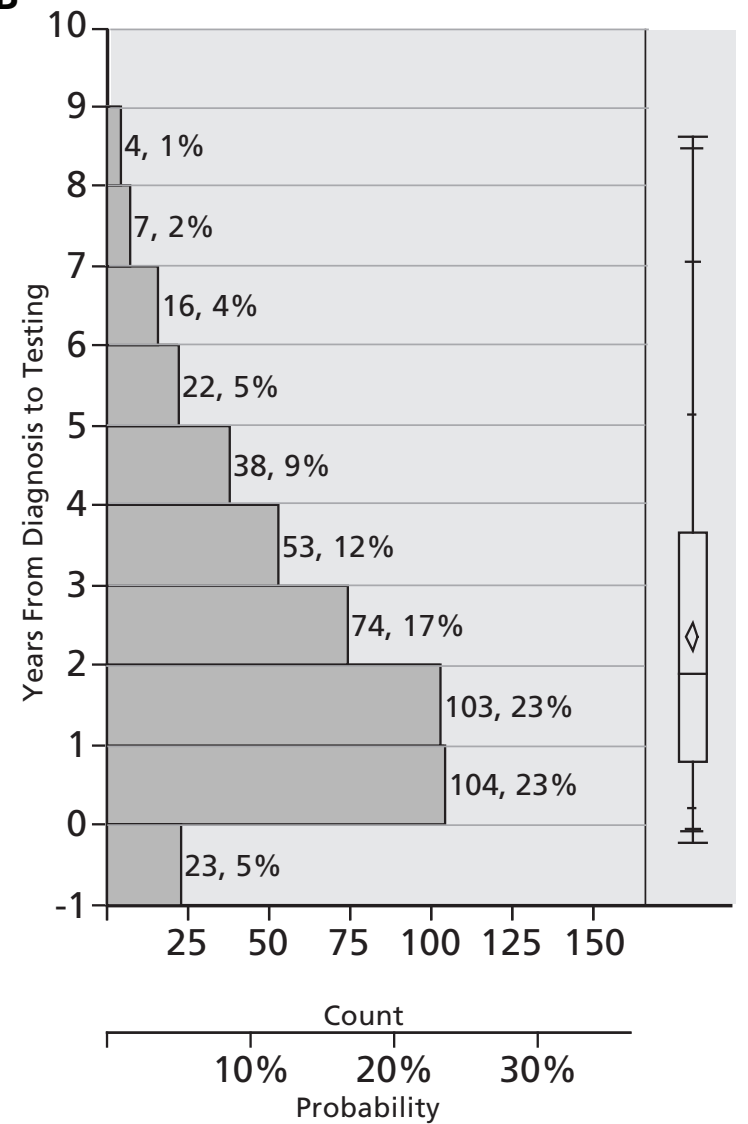

Figure 2 Distribution and timing of all (A) cytogenetic $(n=153)$ and (B) molecular tests $(n=444)$ ordered for monitored patients during follow-up with quantile box plot. 


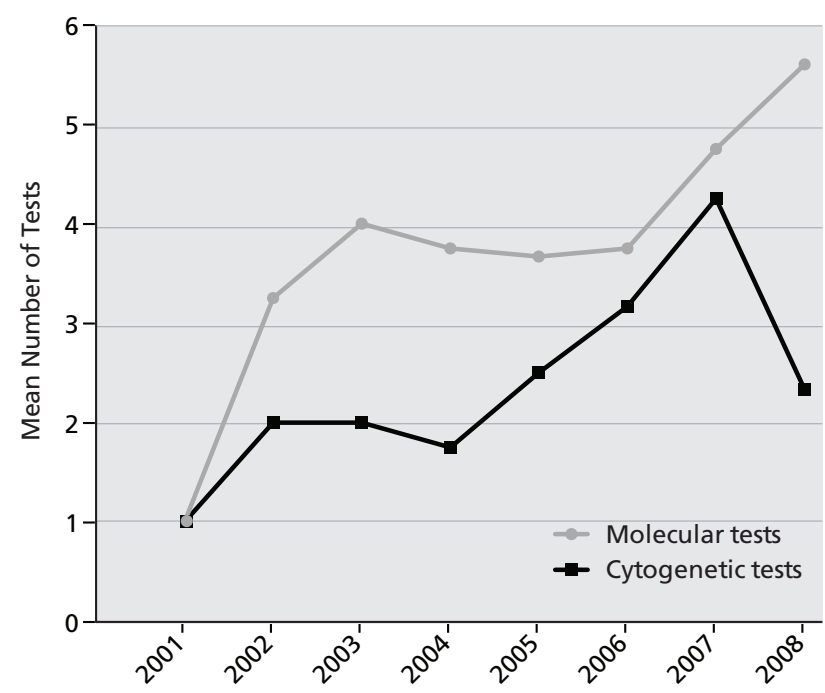

Figure 3 Mean number of molecular and cytogenetic tests ordered within 18 months of imatinib initiation per patient by year of diagnosis.

response or treatment failure with a median time to dose increase of 329 days. In patients diagnosed between 2006 and 2010 ( $n=30 ; 56 \%), 7$ (23\%) transitioned to dasatinib or nilotinib, with a median time to transition of 399 days (range, 180-1046) from diagnosis. The given reasons for this change were suboptimal response or treatment failure $(n=5)$ and ADEs $(n=2)$. Two of these patients were treated with an increased dose of imatinib $(600 \mathrm{mg})$ before transition to dasatinib or nilotinib.

\section{Discussion}

This single-institution observational study of patients with CP-CML treated with first-line imatinib at an NCCN Member Institution corroborates the survival benefit reported in clinical trial results. The IRIS trial ${ }^{1}$ showed an OS rate of $89 \%$ at 5 years, which compares favorably with the value of $88.5 \%$ obtained in the present study and other observational studies. ${ }^{5}$

Use of cytogenetic testing was consistent with guideline recommendations during the study period. Cytogenetic testing was used predominately from the time of diagnosis to 18 months after initiation of imatinib (Figure 2A), which may be explained by the large percentage of patients experiencing a CCyR (73\%) and an MMR (50\%) within 18 months, and therefore cytogenetic testing would not be necessary because of the increased sensitivity of molecular testing. The frequency of cytogenetic

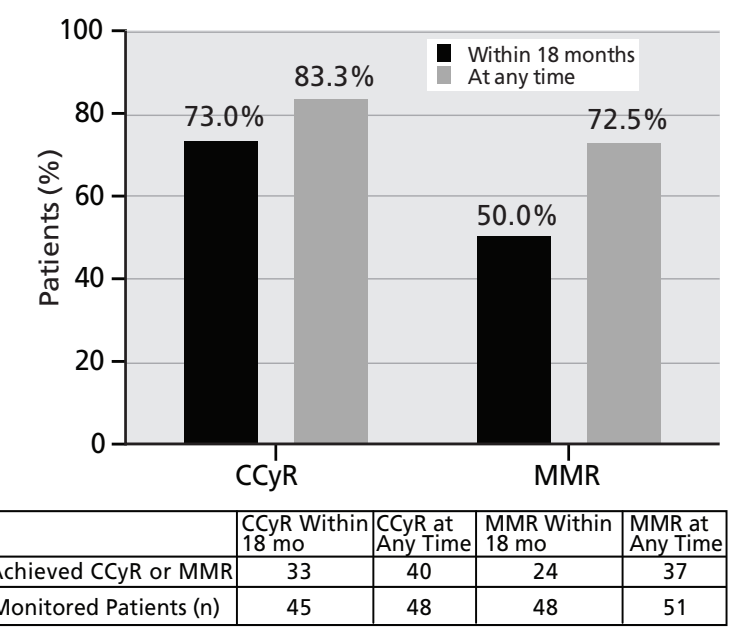

Figure 4 Percentage of monitored patients achieving complete cytogenetic response (CCyR) or major molecular response (MMR) within 18 months or at any time during follow-up.

testing from diagnosis to 18 months after initiation of imatinib increased during the study period from approximately 2 tests per patient diagnosed in 2001 to 2004 to 3 to 4 tests per patient diagnosed in 2005 to 2008. In the period from 2001 to 2004, recommendations for cytogenetic testing of patients on imatinib therapy included testing at the time of diagnosis and subsequently at 3-to 6-month intervals until achievement of CCyR. ${ }^{26}$ In patients diagnosed from 2005 to 2008, the mean number of cytogenetic tests completed increased to 3 to 4 per patient. Reasons for this increase in cytogenetic testing may be explained by the data published during this time correlating obtainment of CCyR and OS benefit, , $27^{27}$ and the generation of monitoring guidelines based on those results. The current NCCN Clinical Practice Guidelines in Oncology (NCCN Guidelines) for CML recommend bone marrow cytogenetic testing at diagnosis, at 3 and 6 months if quantitative PCR (IS) is not available, and at 12 and 18 months if the patient is not experiencing CCyR or MMR (to view the most recent version of these guidelines, visit NCCN.org),${ }^{14}$ which is consistent with practice patterns during the later period of the present study.

Molecular testing was also consistent with guideline recommendations. In the present study, the median number of molecular tests conducted from diagnosis to 18 months after starting therapy was 3.8 (1 test every 4.7 months). During the entire study period, the median number of tests completed per monitored patient was 8.7 , which, based on the me- 
Monitoring and Tolerance of Imatinib in CML

dian follow-up time of 3.14 years, is approximately 1 test every 4.3 months. The mean number of molecular tests conducted from diagnosis to 18 months after initiation of imatinib increased from 1 test per patient diagnosed in 2001 to a consistent 4 tests per patient diagnosed in 2002 to 2006, and an increasing trend was observed for patients diagnosed in 2007 and 2008 (Figure 3). During the period from 2001 to 2006, molecular testing was recommended every 3 to 6 months after achievement of CCyR, and was not recommended at diagnosis. ${ }^{26,28}$ Molecular testing was rapidly adopted and used at the institution during this period. In 2006, the NCCN Guidelines changed the recommendations for molecular testing, to testing at diagnosis and at 3-month intervals unless increasing levels of BCR-ABL1 transcripts were observed. In the present study, the mean number of molecular tests conducted per patient diagnosed in 2007 and 2008 subsequently increased to 4.8 and 5.6 tests per patient (from diagnosis to within 18 months after initiation of imatinib), respectively, which closely approximates the 6 tests recommended at days $0,90,180,270,360$, and 450 leading up to the 18 -month evaluation (540 days). The frequency of all molecular tests conducted in the cohort was consistent over the first 2 years postdiagnosis, but decreased in subsequent years (Figure 2B), which may reflect the duration of follow-up in this study or the practice patterns of clinicians and patients on obtainment of CCyR or MMR. The observations illustrate the effect of national guidelines on practice patterns at a referral cancer center.

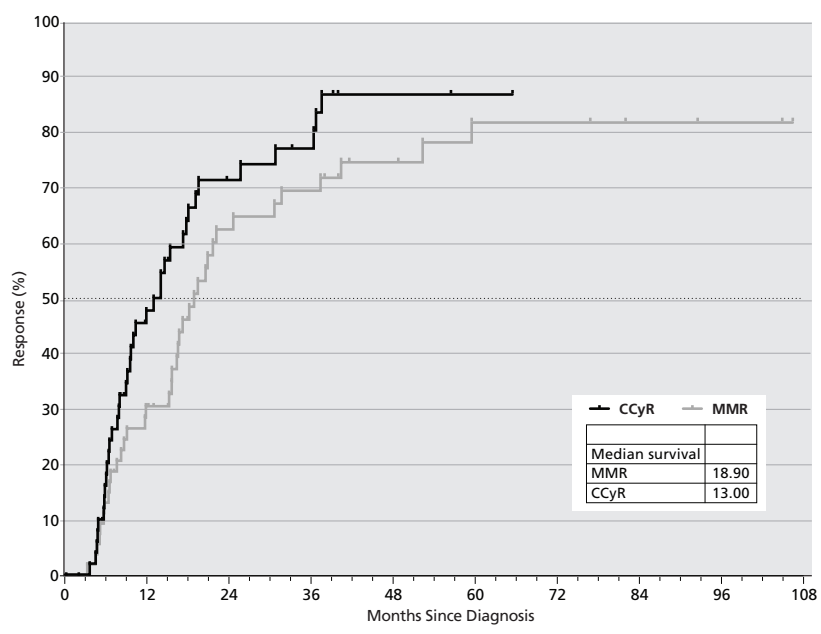

Figure 5 Time to first complete cytogenetic response (CCyR) or major molecular response (MMR) calculated using the Kaplan-Meier method.
In monitored patients, the CCyR and MMR rates approached values observed in clinical trials. ${ }^{1,29}$ In the present study, $73 \%$ of patients experienced a CCyR within 18 months, with a median time from diagnosis of 241 days (Figure 4). An MMR was achieved in fewer patients and with a longer median time to response, as expected. The estimated rate of CCyR at 18 months in the IRIS trial ${ }^{1}$ was $76.2 \%$, similar to the $73.0 \%$ observed in the present cohort. The MMR rate at 18 months with imatinib in the Evaluating Nilotinib Efficacy and Safety in Clinical Trials-Newly Diagnosed Patients (ENESTnd) trial ${ }^{30}$ was $40 \%$ compared with $50 \%$ in this study. These results differ from those observed in nonacademic, community settings, wherein $49 \%$ to $64 \%$ of patients experienced a CCyR at 18 months and $27 \%$ to $47 \%$ achieved an MMR at 18 months. ${ }^{31,32}$

These ADE findings are comparable to those of the IRIS study. Imatinib-associated adverse events occurred in $57 \%$ of patients, which is similar to rates observed in clinical trials and postmarketing experience.,10,29 The discontinuation rate of imatinib therapy because of $\mathrm{ADEs}$ is $18.6 \%$ to $36.0 \%$ in clinical trial experience, ${ }^{1,5,10,29}$ which is also similar to the $19 \%$ observed in the present study. Imatinib dose reductions and discontinuation occurred early in treatment, and a trend toward an increased time to first MMR was observed for those with an ADE compared with those without. Overall, imatinib ADEs occurred early, frequently resulting in dose reductions or discontinuations. Further study is warranted in larger observational cohorts to assess the effect of ADEs on time to MMR.

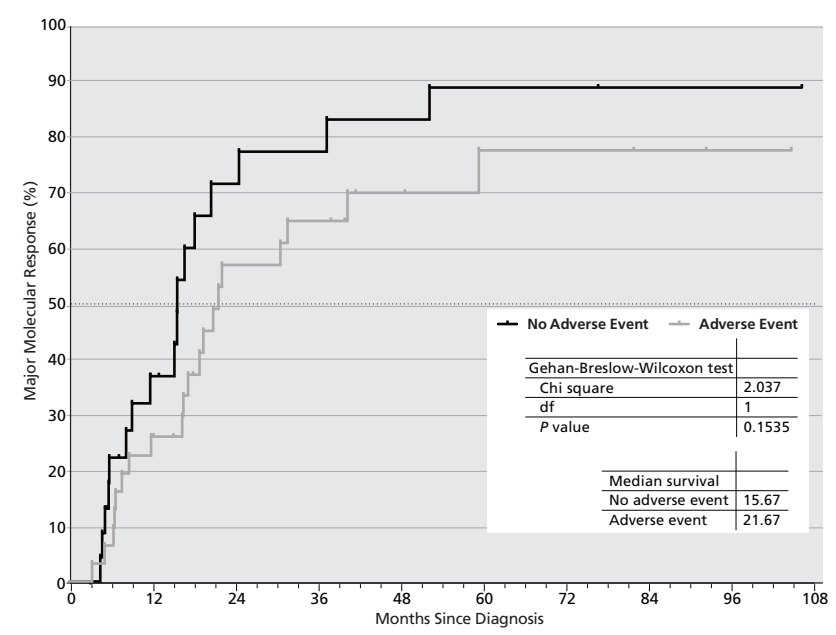

Figure 6 Time to first major molecular response between patients with and without an adverse drug event, calculated using the KaplanMeier method. 


\section{Limitations}

This study is limited by the small sample size and single-institutional setting. In addition, the research outcomes database was based on an EHR, and therefore biomedical records outside of UUHC/HCI EDW were not available. Additionally, this cohort reflects the practice at a specialized hematology clinic in a referral cancer center, focusing on leukemia therapy. Given these study limitations, the results may not be generalizable to the community setting.

\section{Conclusions}

This article reports a single-institutional experience of patients with CP-CML treated with imatinib. The authors were able to confirm the current use of imatinib therapy and the use of cytogenetic and molecular testing to monitor response and associated outcomes in the setting of an NCCN Member Institution or NCI-designated cancer center. These results demonstrate the benefit of institutional adherence to national guidelines for monitoring response to therapy and corroborate findings related to imatinib in key clinical trials.

\section{Acknowledgments}

The authors would like to acknowledge Reed Barney and Brian Oberg for data extraction and management from the University of Utah Enterprise Data Warehouse.

\section{References}

1. O'Brien SG, Guilhot F, Larson RA, et al. Imatinib compared with interferon and low-dose cytarabine for newly diagnosed chronicphase chronic myeloid leukemia. N Engl J Med 2003;348:9941004.

2. Druker BJ, Guilhot F, O'Brien SG, et al. Five-year follow-up of patients receiving imatinib for chronic myeloid leukemia. $\mathrm{N}$ Engl J Med 2006;355:2408-2417.

3. Deininger M, O'Brien SG, Guilhot $F$, et al. International Randomized Study of Interferon Vs STI571 (IRIS) 8-year follow up: sustained survival and low risk for progression or events in patients with newly diagnosed chronic myeloid leukemia in chronic phase (CML-CP) treated with imatinib \{abstract]. Blood 2009;114:Abstract 1126.

4. Efficace F, Cocks K, Breccia M, et al. Time for a new era in the evaluation of targeted therapies for patients with chronic myeloid leukemia: inclusion of quality of life and other patient-reported outcomes. Crit Rev Oncol Hematol 2012;81:123-135.
5. de Lavallade H, Apperley JF, Khorashad JS, et al. Imatinib for newly diagnosed patients with chronic myeloid leukemia: incidence of sustained responses in an intention-to-treat analysis. J Clin Oncol 2008;26:3358-3363.

6. Kantarjian H, Shah NP, Hochhaus A, et al. Dasatinib versus imatinib in newly diagnosed chronic-phase chronic myeloid leukemia. N Engl J Med 2010;362:2260-2270.

7. Saglio G, Kim DW, Issaragrisil S, et al. Nilotinib versus imatinib for newly diagnosed chronic myeloid Leukemia. N Engl J Med 2010;362:2251-2259.

8. Khoury HJ, Cortes JE, Kantarjian HM, et al. Bosutinib is active in chronic phase chronic myeloid leukemia after imatinib and dasatinib and/or nilotinib therapy failure. Blood 2012;119:34033412.

9. Cortes JE, Kantariian H, Shah NP, et al. Ponatinib in refractory philadelphia chromosome-positive leukemias. N Engl J Med 2013;367:2075-2088.

10. Mauro MJ, Deininger MW. Management of drug toxicities in chronic myeloid leukaemia. Best Pract Res Clin Haematol 2009;22:409-429.

11. Marin D, Bazeos A, Mahon FX, et al. Adherence is the critical factor for achieving molecular responses in patients with chronic myeloid leukemia who achieve complete cytogenetic responses on imatinib. J Clin Oncol 2010;28:2381-2388.

12. Ibrahim AR, Eliasson L, Apperley JF, et al. Poor adherence is the main reason for loss of CCyR and imatinib failure for chronic myeloid leukemia patients on long-term therapy. Blood 2011;117:3733-3736.

13. Noens L, van Lierde MA, De Bock R, et al. Prevalence, determinants, and outcomes of nonadherence to imatinib therapy in patients with chronic myeloid leukemia: the ADAGIO study. Blood 2009;113:5401-5411.

14. O'Brien S, Radich JP, Abboud CN, et al. NCCN Clinical Practice Guidelines in Oncology: Chronic Myelogenous Leukemia. Version 3, 2014. Available at: NCCN. org. Accessed June 20, 2014.

15. Marin D, Ibrahim AR, Lucas $C$, et al. Assessment of BCR-ABL1 transcript levels at 3 months is the only requirement for predicting outcome for patients with chronic myeloid leukemia treated with tyrosine kinase inhibitors. J Clin Oncol 2012;30:232-238.

16. D'Hoore W, Bouckaert A, Tilquin C. Practical considerations on the use of the Charlson comorbidity index with administrative data bases. J Clin Epidemiol 1996;49:1429-1433.

17. Data Resource Center: FAQ's. University of Utah Health Sciences Center. Available at: http://uuhsc.utah.edu/drc/faqs. html. Accessed June 20, 2014.

18. Data Resource Center: Home. University of Utah Health Sciences Center. Available at: http://uuhsc.utah.edu/drc/. Accessed June 20, 2014.

19. Data Resource Center: Our Services. University of Utah Health Sciences Center. Available at: http://uuhsc.utah.edu/drc/services. html. Accessed June 20, 2014.

20. The University of Utah Pedigree and Population Resource: Utah population database: overview. Huntsman Cancer Institute. Available at: http://healthcare.utah.edu/ huntsmancancerinstitute/research/updb/. Accessed June 20, 2014.

21. Pulte D, Gondos A, Redanial MT, Brenner H. Survival of patients with chronic myelocytic leukemia: comparisons of estimates from clinical trial settings and population-based cancer registries. Oncologist 2011;16:663-671. 
Monitoring and Tolerance of Imatinib in CML

22. The University of Utah Pedigree and Population Resource: Utah population database: access to data. Huntsman Cancer Institute. Available at: http://healthcare.utah.edu/ huntsmancancerinstitute/research/updb/access.php. Accessed June 20, 2014.

23. The University of Utah Pedigree and Population Resource: Utah population database: medical records. Huntsman Cancer Institute. Available at: http://healthcare.utah.edu/ huntsmancancerinstitute/research/updb/data/medical-records. php. Accessed June 20, 2014.

24. The University of Utah Pedigree and Population Resource: Utah population database: other records. Huntsman Cancer Institute. Available at: http://www.huntsmancancer.org/research/sharedresources/utah-population-database/other-records. Accessed June 20, 2014.

25. The University of Utah Pedigree and Population Resource: Utah population database: vital records. Huntsman Cancer Institute. Available at: http://www.huntsmancancer.org/research/sharedresources/utah-population-database/vital-records. Accessed June 20, 2014.

26. Tefferi A, Dewald GW, Litzow ML, et al. Chronic myeloid leukemia: current application of cytogenetics and molecular testing for diagnosis and treatment. Mayo Clin Proc 2005;80:390 402 .
27. Kantarjian HM, O’Brien S, Cortes JE, et al. Complete cytogenetic and molecular responses to interferon- $\alpha$-based therapy for chronic myelogenous leukemia are associated with excellent long-term prognosis. Cancer 2003;97:1033-1041.

28. Baccarani M. Evolving concepts in the management of chronic myeloid leukemia: recommendations from an expert panel on behalf of the European LeukemiaNet. Blood 2006;108:18091820.

29. Kantarjian HM, Hochhaus A, Saglio G, et al. Nilotinib versus imatinib for the treatment of patients with newly diagnosed chronic phase, Philadelphia chromosome-positive, chronic myeloid leukaemia: 24-month minimum follow-up of the phase 3 randomised ENESTnd trial. Lancet Oncol 2011;12:841-851.

30. Larson RA, Hochhaus A, Hughes TP, et al. Nilotinib vs imatinib in patients with newly diagnosed Philadelphia chromosomepositive chronic myeloid leukemia in chronic phase: ENESTnd 3-year follow-up. Leukemia 2012;26:2197-2203.

31. Lucas CM, Wang L, Austin GM, et al. A population study of imatinib in chronic myeloid leukaemia demonstrates lower efficacy than in clinical trials. Leukemia 2008;22:1963-1966.

32. Francis S, Lucas C, Lane S, et al. A population study showing that the advent of second generation tyrosine kinase inhibitors has improved progression-free survival in chronic myeloid leukaemia. Leuk Res 2013;37:752-758. 